\title{
MEMS ACTUATION FOR A CONTINUOUSLY TUNABLE OPTICAL BUFFER
}

\author{
N. Podoliak ${ }^{1}$, W. H. Ng ${ }^{2}$, H. Liu ${ }^{2}$, A. J. Kenyon ${ }^{2}$, W. Stewart ${ }^{1}$, P. Horak ${ }^{1}$ \\ ${ }^{1}$ Optoelectronics Research Centre, University of Southampton, UK \\ ${ }^{2}$ Department of Electronic \& Electrical Engineering, University College London, UK \\ E-mail: N.Podoliak@ soton.ac.uk
}

\begin{abstract}
We present our work towards development of an optical buffer based on the InP platform that consists of two suspended coupled optical waveguides. A continuously tunable delay in the propagation time can be achieved by varying the spacing between the waveguides using MEMS actuation. Up to $100 \%$ variation of the delay time with a driving voltage of $3 \mathrm{~V}$ is predicted.
\end{abstract}

\section{INTRODUCTION}

The main function of an optical buffer in telecommunication networks is to retain data in an optical format for a desired period or in other words, to introduce a controllable delay to an optical signal. In our previous work we demonstrated the possibility of continuously variable optical buffering in a dual suspended optical waveguide system where the delay is achieved by mechanical reconfiguration of the system [1]. Here we present our work towards a realization of this scheme in InP including modelling of optical and mechanical properties of the waveguides, design of electrodes for the buffer actuation, simulation of the buffer performance and first steps towards its fabrication.

\section{BUFFER OPERATION PRINCIPLE}

The schematic design of the optical buffer is shown in Figure 1. The optical part of the buffer consists of two rectangular waveguides made of high index material and suspended in air. The waveguides are separated by a small air gap and remain optically coupled. The optimized waveguide dimensions are $\sim 195 \times 300 \mathrm{~nm}$. When the two waveguides are close together, a large fraction of light is confined within the waveguides, as shown in the inset in Figure 1, leading to a large effective group index. When the waveguides are pulled apart, the optical field expands more in the air, resulting in a lower group index and hence a faster propagation of signals. An optical delay variation of $100 \%$ can be achieved when the separation between the two waveguides changes by $\sim 500 \mathrm{~nm}$, see below.

The waveguides are supported by pillars connected from the side as shown in Figure 1. The alternating positions of the pillars enable the initially straight waveguides to bend in a way to create a structure with more or less uniform separation over the whole length. A distance between two pillars of $100 \mu \mathrm{m}$ is predicted to ensure sufficient flexibility of the waveguides and enables large enough mechanical oscillation frequency $(\sim 75-100 \mathrm{kHz})$ to reduce vibration noise.

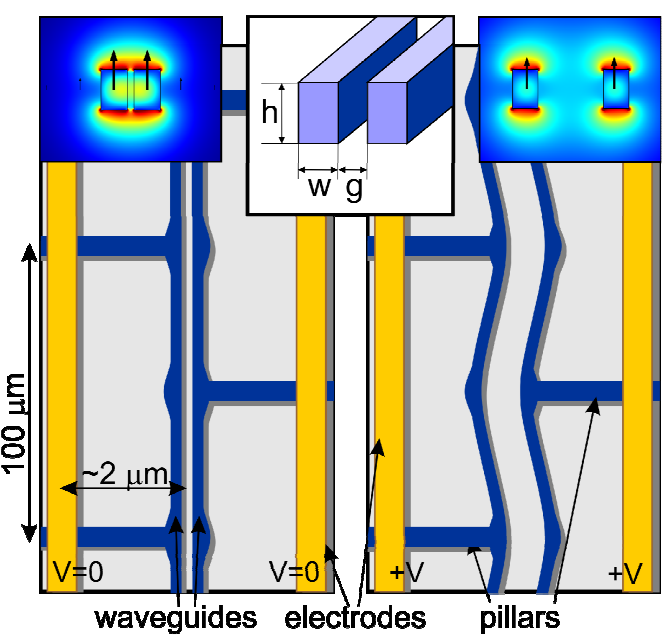

Figure 1. Schematic of the optical buffer (top view, not to scale). Insets: coupled optical waveguides of height $h$ and width $w$ separated by distance $g$ (center); electric field profile of the fundamental TE mode at $50 \mathrm{~nm}$ (left) and 500nm (right) gap between waveguides.

\section{ELECTROSTATIC ACTUATION}

There are two possible actuation principles based on (i) reducing the gap between two waveguides with increasing voltages or (ii) separating the initially closely placed waveguides. The first operation principle suffers from a pull-in instability, where elastic forces cannot counteract electrostatic forces beyond a certain threshold, which is likely to result in sticking of the waveguides. We thus concentrate on implementing the second actuation mechanism where sticking of the waveguides is avoided.

Variation of the distance between two waveguides can be achieved using MEMS techniques. We simulated two different electrostatic mechanisms for the optical buffer actuation, which we call "passive" and "active". In the passive method, the waveguide actuation is achieved via electrostatic dipole forces without direct electrical contacts to the waveguides [2]. The modelled geometry consists of two continuous electrodes along the waveguides placed at 
a distance of around $2 \mu \mathrm{m}$ to eliminate interaction with the guided optical field (as schematically shown in Figure 1). The electrodes are charged with the same polarity and the bottom substrate $(\sim 3-4 \mu \mathrm{m}$ below the waveguides) is grounded. This generates an electric field with a significant field gradient around the waveguides, which resembles a quadrupole field configuration discussed recently for MEMS in optical fibers [3]. The field polarizes the waveguides, which are thus pulled towards regions of stronger field, increasing the separation of the waveguides. In the modelled geometry a gap variation from $50 \mathrm{~nm}$ up to $500 \mathrm{~nm}$ is predicted with a driving voltage of $\sim 45 \mathrm{~V}$ (see inset in Figure 2), above which a pull-in instability occurs and the waveguides collapse onto the electrodes.

The second, "active" method relies on voltages being applied directly to the waveguides, so no electrodes are needed. Repulsion between the initially closely spaced waveguides is achieved by applying the same voltage polarity to them, while the bottom substrate is again grounded. The direct interaction between charged waveguides generates stronger forces. Our calculations show that a driving voltage of only $3 \mathrm{~V}$ is required to actuate the buffer, which is much lower than that required for the passive method. Moreover, no pull-in instability exists with this method. Up to $100 \%$ variation of the group index is shown in Figure 2, which corresponds to variation of a delay time by $\sim 10 \mathrm{ps}$ in a $1 \mathrm{~mm}$ long device.

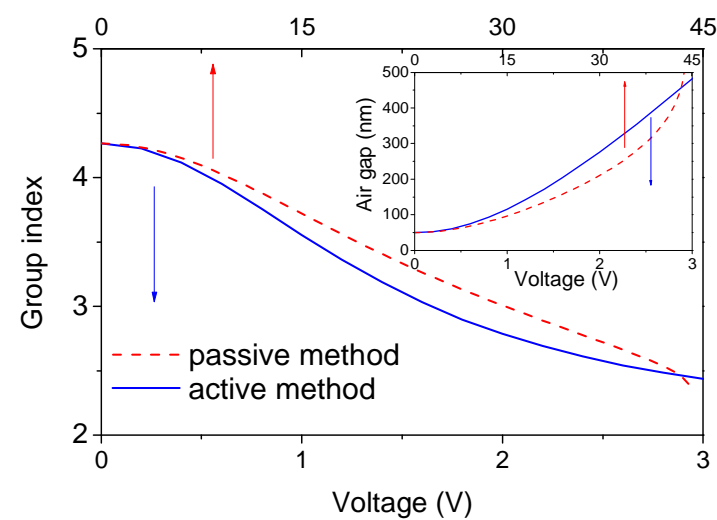

Figure 2. Variation of average group index depending on voltage. Inset: variation of average gap depending on voltage applied to the waveguides.

To realize the active method, the waveguide material has to be doped, which leads to increased free-carrier absorption (FCA) compared to the passive method. However, the FCA losses for n-type doped InP with a doping concentration of $n=5 \times 10^{17} \mathrm{~cm}^{-3}$ are estimated to be $\sim 2.5 \mathrm{~dB} / \mathrm{cm}$, which is of the order of expected propagation losses due to sidewall roughness and should therefore be acceptable for the device.

\section{FABRICATION}

The device is fabricated in InP, which will allow for broadband operation and active loss compensation. The initial substrate from which the device is fabricated consists of $\sim 3 \mu \mathrm{m}$ thick $\operatorname{In}_{0.53} \mathrm{Ga}_{0.47}$ As layer (sacrificial layer) grown onto $\mathrm{InP}$ substrate and $300 \mathrm{~nm}$ thick layer of InP (waveguide and electrode layer) deposited on the top.

The electrodes, waveguides and pillar supports were patterned on the InP layer by electron beam lithography (Raith150-TWO). Gold electrodes were deposited by thermal evaporation and lift off in acetone. The patterned structure was etched by reactive ion etching (RIE) with a cyclic methane-hydrogen/oxygen plasma. The resulting etched structure has a vertical sidewall angle better than 80 degrees. The waveguide layer was released by etching the $\mathrm{In}_{0.57} \mathrm{Ga}_{0.43}$ As sacrificial layer with $\mathrm{HF}: \mathrm{H}_{2} \mathrm{O}_{2}: \mathrm{H}_{2} \mathrm{O}(1: 1: 8)$ solution. The resulting free standing waveguide is shown in Figure 3. Work on further optimization of the fabrication and demonstration of the optical buffer is ongoing.

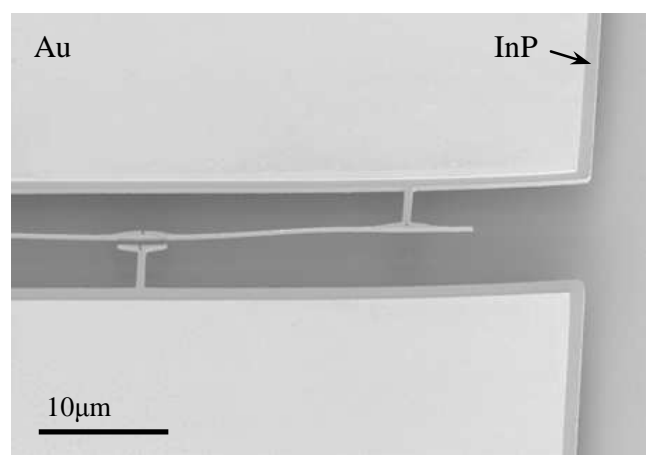

Figure 3. Free standing waveguide and pillars after etching the $\mathrm{In}_{0.57} \mathrm{Ga}_{0.43} \mathrm{As}$ sacrificial layer. Only one waveguide is developed.

This work was supported by the EPSRC "Continuously tunable optical buffer" project (EP/J012874/1 and EP/J012823/1).

\section{REFERENCES}

[1] P. Horak, W. Stewart, W. H. Loh, "Continuously tunable optical buffer with a dual silicon waveguide design" Opt. Express, vol. 19, pp. 12456-12461, 2011.

[2] M. C. Lee and M. C. Wu, "Tunable coupling regimes of silicon microdisk resonators using MEMS actuators" Opt. Express, vol. 14, pp. 4703-4712, 2006.

[3] N. Podoliak, Z. Lian, W. H. Loh, P. Horak, "Design of dual-core optical fibers with NEMS functionality" Opt. Express, vol. 22, pp. 1065-1076, 2014. 